\title{
Warfarina y anticoagulantes orales directos. Revisión sistemática y metanálisis sobre estudios de no inferioridad en fibrilación auricular y trombosis venosa profunda.
}

\author{
Gilberto Viæcaíno ${ }^{1,2}$, Jesús Weir Medina ${ }^{3}$, Jesús Quintero ${ }^{1}$ y Jennifer Viæcaíno-Carruyo ${ }^{4}$ \\ ${ }^{1}$ Instituto de Investigaciones Clínicas "Dr. Américo Negrette”, Facultad de Medicina, \\ Universidad del Zulia, Maracaibo, Venezuela. \\ ${ }^{2}$ Instituto de Investigación. Universidad Técnica Manabí, Portoviejo. Ecuador. \\ ${ }^{3}$ Instituto Hematológico de Occidente, Maracaibo, Venezuela. \\ ${ }^{4}$ Editorial Médica Colombiana, Medellín, Colombia.
}

Palabras clave: warfarina; anticoagulantes anti-vitamina $\mathrm{K}$; anticoagulantes orales directos; fibrilación auricular; trombosis venosa profunda; estudios de no inferioridad.

Resumen. Los anticoagulantes orales directos (ACODs), se han convertido en una alternativa de anticoagulación en fibrilación auricular (FA) y en trombosis venosa profunda (TVP). Los estudios de no inferioridad entre los ACODs y la warfarina, están diseñados para demostrar que los primeros mantienen al menos la misma eficacia y mejor seguridad que la warfarina. Se realizó una revisión sistemática y metanálisis de ensayos aleatorios controlados en FA (4 estudios) y TVP (6 estudios). El evento primario fue el ictus isquémico y embolismo sistémico) en FA y la trombosis venosa recurrente para TVP, y la hemorragia mayor para seguridad en ambas patologías. En FA, el análisis mostró equivalencia entre los ACODs y la warfarina, el resultado combinado evidenció una reducción significativa del riesgó de 24\% [RR (IC95\%)=0,76 (0,69-0,84), p<0,0001]. En el análisis de seguridad, en todos los estudios no hubo diferencias con warfarina y el resultado combinado mostró una reducción no significativa del riesgo del 14\% [RR (IC95\%)=0,86 (0,76$1,01), \mathrm{p}=0,058]$. Para TVP recurrente, todos los estudios mostraron criterios de no inferioridad respecto a la warfarina pero sin uniformidad; el resultado combinado no obtuvo significación estadística [RR $($ IC95\%) $=0,93(0,79-1,08), p=0,372]$. El análisis de seguridad indicó que los ACODs fueron superiores a la warfarina, con una reducción siǵnificativa del riesǵo de hemorragia $(42 \%)$ [RR (IC95\%) =0,58 (0,420,79), p<0,0001]. En conclusión, estos resultados señalan que los ACODs son una buena alternativa para el uso terapéutico en FA y TVP, con una eficacia similar a la warfarina pero una ligera ventaja en la reducción del riesgo hemorrágico.

Autor de correspondencia: Gilberto Vizcaíno. Instituto de Investigaciones Clínicas "Dr. Américo Negrette", Facultad de Medicina, Universidad del Zulia, Maracaibo, Venezuela. Correo electrónico: gilvizcaino@gmail.com 


\title{
Warfarin and direct oral anticoagulants: a systematic review on non-inferiority studies in atrial fibrillation and deep venous thrombosis.
}

\author{
Invest Clin 2020; 61 (1): 73-90
}

Key words: warfarin; anti-vitamin K anticoagulants; new oral anticoagulants; atrial fibrillation; deep vein thrombosis; non-inferiority studies.

\begin{abstract}
New oral anticoagulants (NOACs) have become an alternative for patients receiving long-term anticoagulation in atrial fibrillation (AF) and deep vein thrombosis (DVT). Studies of non-inferiority between NOACs and warfarin are designed to demonstrate that the former maintain at least the same efficacy and better safety in hemorrhagic events than warfarin. A systematic review and meta-analysis of randomized controlled trials was conducted in atrial fibrillation (AF) and deep vein thrombosis (DVT). The primary event was stroke (ischemic stroke and systemic embolism) for AF, recurrent venous thrombosis for DVT and the safety event was major bleeding for both pathologies. In AF, the analysis showed equivalence and significant reduction of NOACs compared to warfarin, with a $24 \%$ reduction in the relative risk $[\mathrm{RR}(\mathrm{IC95 \% )}=0.76(0.69-0.84)$, $\mathrm{p}<0.001]$. In the safety analysis, all studies did not reveal differences with warfarin and the overall result showed a non-significant risk reduction of $14 \%$ [RR $(\mathrm{IC95 \%})=0.86(0.76-1.01), \mathrm{p}=0.058]$. For DVT, all studies showed non-inferiority criteria in comparison with warfarin [RR $(\mathrm{IC95 \%})=0.93(0.79-1.08), \mathrm{p}=0,372]$. The safety analysis indicated superiority of NOACs over warfarin in all studies. The combined result revealed that there was a significant reduction of the risk of bleeding (42\%) in favor of NOACs compared to warfarin [RR (IC95\%) $=0.58$ $(0.42-0.79), \mathrm{p}<0.0001]$. In conclusion, these results showed that NOACs are a good alternative for therapeutic use in AF and DVT, with similar efficacy on warfarin but with a slight advantage in the reduction of hemorrhagic risk.
\end{abstract}

Recibido: 01-10-2019 Aceptado: 23-01-2020

\section{INTRODUCCIÓN}

La warfarina es el principal anticoagulante oral tipo anti-vitamina $\mathrm{K}$ (AVK), cuyo uso se ha extendido por más de 60 años para la prevención primaria y secundaria de enfermedades tromboembólicas, especialmente en patologías de tipo cardiovascular (fibrilación auricular, insuficiencia cardíaca congestiva, miocardiopatía isquémica, etc.), en TVP, en embolismo pulmonar y en la trom- bosis asociada al cáncer. Desde el año 1948, entró en escena la aplicación terapéutica de la warfarina como anticoagulante oral pero su uso generalizado ocurrió hacia el año $1954(1,2)$. Esta era fue dominada por los AVK, la heparina no fraccionada y las heparinas de bajo peso molecular, como agentes anticoagulantes en el tratamiento de enfermedades tromboembólicas. A principios del siglo XXI, comenzó la administración terapéutica de los nuevos anticoagulantes orales 
directos, caracterizados en dos grupos por su mecanismo de acción: antitrombínicos (dabigatran) y anti factor Xa (rivaroxaban, apixaban, edoxaban). Las acepciones de "gatranes" y "xabanes" para identificar estos dos grupos fueron acuñadas por PérezRequejo y col. (3), en su excelente revisión sobre los ACODs (anticoagulantes orales directos). El empleo de los ACODs trajo una ventaja sobre la warfarina y es el no necesitar control del laboratorio clínico sobre su efecto anticoagulante, lo que si es necesario con el uso de la warfarina que necesita controlar su efecto a través del cálculo del INR (Razón Internacional Normalizada) para establecer un rango terapéutico entre 2 y 3 , rango que señala generalmente una anticoagulación apropiada para la mayoría de los casos, aunque estudios realizados han señalado que un INR más bajo puede ser efectivo en la prevención de eventos trombóticos (4-6). Las otras diferencias entre AVK y ACODs estriban en que los primeros poseen una variable interacción con drogas y alimentos (7) (Tabla I) y necesitan un tiempo entre $24 \mathrm{y}$ 48 horas, para lograr el rango terapéutico; por otra parte, cuando se necesita interrumpir el tratamiento, su efecto anticoagulante puede perdurar por horas. Adicionalmente, es necesario establecer el tiempo en rango terapéutico o TRT (TTR:Time in Terapeutic Range) (8) para mantener su efectividad y seguridad, es decir el control terapéutico de los AVK especialmente al inicio es un poco complicado y debe ser individualizado, al respecto se ha reportado que el mantenimiento optimo del TRT influye más en la seguridad que en la efectividad del tratamiento (9).

Las ventajas de los ACODs sobre los AVK, estarían en que su acción anticoagulante es puntual (contra la trombina o el FXa) y rápida para lograr su vida media (6-12 hrs), lo que representa una ventaja si se requiere su interrupción y poseen poca interacción con drogas $(10,11)$ y alimentos $(12)$. La Tabla II representa las interacciones con drogas de los ACODs a través de la inhibición o inducción del citocromo P450 3A4 (CYP3A4) y la glicoproteína P (P-gp) transportadora, los cuales se expresan en la mucosa intestinal y presentan una barrera para la administración oral de fármacos, por ejemplo, el dabigatran y el rivaroxaban no deberían ser administrados con inhibidores de P-gp y déficit en la función renal ya que potencian el riesgo hemorrágico con esta interacción $(10,11,13)$.

TABLA I

INTERACGIONES FARMACOLÓGICAS CON INHIBIDORES DE LA VITAMINA K (WARFARINA)

\begin{tabular}{|c|c|c|c|c|c|}
\hline $\begin{array}{l}\text { Antibióticos/ } \\
\text { Antimicóticos }\end{array}$ & $\begin{array}{l}\text { Drogas de efecto } \\
\text { Cardiovascular }\end{array}$ & $\begin{array}{l}\text { Drogas de efecto } \\
\text { Antinflamatorio }\end{array}$ & $\begin{array}{l}\text { Drogas de efecto } \\
\text { sobre Sistema } \\
\text { Nervioso Central }\end{array}$ & $\begin{array}{l}\text { Drogas de efecto } \\
\text { Gastrointestinal }\end{array}$ & Otros \\
\hline
\end{tabular}

Potenciadores del efecto anticoagulante

Clotrimazol Amiodarona

Ciproflaxacina Propafenona

Eritromicina Propranolol

Tetraciclina Sulfinpirazona Aspirina

Isoconazol Fibratos Fenilbutazona

Metronidazole Sinvastatina Piroxicam

Cimetidina Tamoxifen

Omeprazol Danazol

Fluconazol Aspirina

Miconazol Ticlopidina

Inhibidores del efecto anticoagulante

Rifampicina A Colestiramina

Nafcilina A

Carbamazepina Sucralfato

Vitamina K

Modificado y adaptado de Pengo V (7).

Vol. 61(1): 73 - 90, 2020 
TABLA II

ANTICOAGULANTES ORALES DIRECTOS E INTERACCIONES CON DROGAS

\begin{tabular}{|c|c|c|}
\hline ACODs & $\begin{array}{l}\text { Inhibidores P-gp transporte } \\
\text { y CYP3A4 (aumentan el efecto } \\
\text { anticoagulante) }\end{array}$ & $\begin{array}{l}\text { Inductores P-g p transporte } \\
\text { y CYP3A4 (disminuyen el efecto } \\
\text { anticoagulante) }\end{array}$ \\
\hline Dabigatran & $\begin{array}{l}\text { Ketoconazol } \\
\text { Itraconazol } \\
\text { Dronedarona }\end{array}$ & Rifampicina \\
\hline Apixaban & $\begin{array}{l}\text { Ketoconazol } \\
\text { Itraconazol } \\
\text { Ritonavi }\end{array}$ & Rifampicina \\
\hline Edoxaban & $\begin{array}{l}\text { Quinidina } \\
\text { Verapamilo } \\
\text { Amiodarona } \\
\text { Dronedarona } \\
\text { Ketoconazol } \\
\text { Eritromicina }\end{array}$ & Rifampicina \\
\hline Rivaroxaban & $\begin{array}{l}\text { Ketoconazol } \\
\text { Itraconazol } \\
\text { Lopinavir } \\
\text { Ritonavir } \\
\text { Indinavir } \\
\text { Conivaptan } \\
\end{array}$ & $\begin{array}{l}\text { Carbamazepine, } \\
\text { Fenitoina } \\
\text { Rifampicina }\end{array}$ \\
\hline
\end{tabular}

Modificado y adaptado de Xiong Q (10); Hellwig T (11).

CYP3A4: Citocromo P3A4, P-gp: ǵlicoproteína p transportadora.

En razón de lo anteriormente expuesto, las grandes preguntas que nos hacemos son las siguientes ¿Pueden los ACODs reemplazar definitivamente a la warfarina como tratamiento de elección o de primera línea para las enfermedades de tipo tromboembólico? Y la segunda pregunta es: ¿Existe evidencia científica relevante en el balance beneficioriesgo de que los ACODs son superiores a la warfarina? Para contestar estas preguntas, en primer lugar, habría que basarse en la Medicina Basada en la Evidencia, cuyos fundamentos asocian la experiencia clínica del médico (juicio crítico), la evidencia científica encontrada y los valores preferenciales del paciente para la toma de una decisión clínica efectiva. La segunda pregunta es respondida en los términos que conocemos como estudios de no inferioridad, para demostrar en la búsqueda de la efectividad y seguridad, si los ACODs son superiores a la warfarina o son no inferiores, es decir, si poseen al menos la misma efectividad y seguridad que la warfarina (14-17). La FA es la causa de arritmia cardíaca más común y su consecuencia principal es el ACV isquémico (ictus) y el embolismo sistémico, su tasa de mortalidad alcanza el $24 \%$ cuando no se recibe tratamiento $(18,19)$. La TVP es una de las principales causas de morbimortalidad para trombosis provocada y no provocada y su tratamiento principal es la anticoagulación parenteral con heparinas no fraccionada o de bajo peso molecular; este tratamiento es combinado con el cambio a anticoagulación oral con warfarina para evitar la trombosis venosa recurrente, no obstante, el beneficio/riesgo se ve opacado por la presencia de hemorragia mayor durante el tratamiento (20). Revisiones Sistemáticas publicadas acerca de ACODs y warfarina muestran heterogeneidad porque incluyen estudios fase 
II (generalmente pocos participantes), estudios fase III (buen número de pacientes), no son enfocadas a criterios de no inferioridad, y concluyen que hay una tendencia de beneficio general de los ACODs en comparación con los AVK, con reducción del riesgo de hemorragiia $(21,22)$. En el caso de TVP algunas revisiones incluyeron metanálisis que demostraron similitud de efecto entre ACODs y AVK en el riesgo de recurrencia, aunque los ACODs (rivaroxaban) poseían menor riesgo de hemorragia (23).

Esta revisión sistemática tiene el propósito de realizar metanálisis de los diferentes estudios de no inferioridad entre los ACODs y la warfarina como control activo, enfocándose solo en dos patologías donde se han publicado mayoritariamente estudios de no inferioridad fase III entre los ACODs y la warfarina: la FA de origen no valvular y la TVP.

\section{METODOLOGÍA}

\section{Estrategias de búsqueda}

La búsqueda bibliográfica se realizó a través de PubMed de MEDLINE y Google Scholar bajo la siguiente terminología
MESH (Medical Subject Headings): "direct oral anticoagulants, warfarin, ribaroxaban, apixaban, edoxaban, dabigatran, controlled randomised studies, atrial fibrillation, stroke and systemic embolism" usando como conector AND para el término "Non-inferiority studies"). La terminología de búsqueda MESH para TVP fue la siguiente: "warfarin, direct oral anticoagulants, dabigatran, rivaroxaban, apixaban, edoxaban, deep venous thrombosis, thrombophlebitis, venous post thrombotic syndrome, venous thrombotic recurrence, pulmonar embolism, controlled randomised studies and non-inferiority studies". Para ambas patologías la búsqueda incluyó un lapso de quince años (2005-2019).

Según las estrategias de búsqueda se identificaron 4 estudios de no inferioridad en FA que reunían los criterios de inclusión: RE-LY (24), ARISTOTLE (25), ROCKET-AF (26) y ENGAGE-TIMI 48 (27) y 6 estudios de no inferioridad para TVP: AMPLIFY (28), EINSTEIN (29), EINSTEIN-PE (30), RECOVER II (31), HOKUSAI-VTE (32) y RE-MEDY (33), los cuales se describen en las Tablas III y IV, respectivamente. Los criterios para la selección de los estudios, fueron: estudios

TABLA III

ANTICOAGULANTES ORALES DIRECTOS Y FIBRILAGIÓN AURICULAR NO VALVULAR. ESTUDIOS DE NO INFERIORIDAD CON WARFARINA COMO CONTROL ACTIVO

\begin{tabular}{|c|c|c|c|c|c|}
\hline $\begin{array}{l}\text { Estudio } \\
\text { (año) (ref.) }\end{array}$ & ACOD & $\begin{array}{l}\text { Duración } \\
\text { del estudio }\end{array}$ & $\begin{array}{l}\mathrm{N}^{\circ} \text { total de pacientes } \\
\mathrm{ACOD} / \text { Warfarina }\end{array}$ & $\begin{array}{l}\text { Evento } \\
\text { primario }\end{array}$ & $\begin{array}{l}\text { Evento } \\
\text { adverso }\end{array}$ \\
\hline $\begin{array}{l}\text { RE-LY* } \\
(2010)(24)\end{array}$ & $\begin{array}{l}\text { Dabigatran } \\
\text { (110-150mg/ } \\
\text { BID) }\end{array}$ & 2 años & $\begin{array}{l}18113 \\
(6015-6076) / 6022\end{array}$ & $\begin{array}{l}\text { Ictus, } \\
\text { embolismo } \\
\text { sistémico }\end{array}$ & $\begin{array}{l}\text { Hemorragia } \\
\text { mayor }\end{array}$ \\
\hline $\begin{array}{l}\text { ARISTOTLE } \\
(2011)(25)\end{array}$ & $\begin{array}{l}\text { Apixaban } \\
\text { (5mg BID) }\end{array}$ & 3,3 años & $\begin{array}{l}18201 \\
9120 / 9081\end{array}$ & $\begin{array}{l}\text { Ictus, } \\
\text { embolismo } \\
\text { sistémico }\end{array}$ & $\begin{array}{l}\text { Hemorragia } \\
\text { Mayor }\end{array}$ \\
\hline $\begin{array}{l}\text { ROCKET-AF } \\
\text { (2011) (26) }\end{array}$ & $\begin{array}{l}\text { Rivaroxaban } \\
\text { (20mg OD) }\end{array}$ & 2 años & $\begin{array}{l}14264 \\
7131 / 7133\end{array}$ & $\begin{array}{l}\text { Ictus, } \\
\text { embolismo } \\
\text { sistémico }\end{array}$ & $\begin{array}{l}\text { Hemorragia } \\
\text { mayor }\end{array}$ \\
\hline $\begin{array}{l}\text { ENGAGE-AF-TIMI } \\
48 \text { (2013) (27) }\end{array}$ & $\begin{array}{l}\text { Edoxaban } \\
\text { (30-60mg OD) }\end{array}$ & 2,8 años & $\begin{array}{l}21105 \\
(7035-7034) / 7036\end{array}$ & $\begin{array}{l}\text { Ictus, } \\
\text { embolismo } \\
\text { sistémico }\end{array}$ & $\begin{array}{l}\text { Hemorragiia } \\
\text { mayor }\end{array}$ \\
\hline
\end{tabular}

"RE-LY corrección del trabajo original publicado en el 2009.

Vol. 61(1): 73 - 90, 2020 
TABLA IV

ANTICOAGULANTES ORALES DIRECTOS Y TROMBOSIS VENOSA PROFUNDA. ESTUDIOS DE NO INFERIORIDAD CON WARFARINA COMO CONTROL ACTIVO

\begin{tabular}{|c|c|c|c|c|c|}
\hline $\begin{array}{l}\text { Estudio } \\
\text { (año) (ref) }\end{array}$ & ACOD & $\begin{array}{l}\text { Duración } \\
\text { del estudio }\end{array}$ & $\begin{array}{l}\mathrm{N}^{\circ} \text { de pacientes } \\
\text { ACOD/Warfarina/ } \\
\text { Total }\end{array}$ & $\begin{array}{l}\text { Evento } \\
\text { Primario }\end{array}$ & $\begin{array}{l}\text { Evento } \\
\text { adverso }\end{array}$ \\
\hline $\begin{array}{l}\text { AMPLIFY } \\
(2013)(28)\end{array}$ & $\begin{array}{l}\text { Apixaban } \\
(10 \mathrm{mg} \text { OD x } 1 \\
\text { semana, luego } \\
5 \mathrm{mg} \text { OD) }\end{array}$ & 3 años & $2609 / 2635 / 5244$ & TVP recurrente & $\begin{array}{l}\text { Hemorragia } \\
\text { mayor }\end{array}$ \\
\hline $\begin{array}{l}\text { EINSTEIN } \\
(2010)(29)\end{array}$ & $\begin{array}{l}\text { Rivaroxaban } \\
(15 \mathrm{mg} \text { BID x } 3 \\
\text { semanas, luego } \\
\text { 20mg OD) }\end{array}$ & 2,5 años & $1718 / 1715 / 3433$ & TVP recurrente & $\begin{array}{l}\text { Hemorragia } \\
\text { mayor }\end{array}$ \\
\hline $\begin{array}{l}\text { EINSTEIN-PE } \\
(2012)(30)\end{array}$ & $\begin{array}{l}\text { Rivaroxaban } \\
(15 \mathrm{mg} \text { BID x } 3 \\
\text { semanas, luego } \\
20 \mathrm{mg} \text { OD) }\end{array}$ & 3 años & $2419 / 2413 / 4832$ & TVP-recurrente & $\begin{array}{l}\text { Hemorragia } \\
\text { mayor }\end{array}$ \\
\hline $\begin{array}{l}\text { RECOVER II } \\
(2013)(31)\end{array}$ & $\begin{array}{l}\text { Dabigatran } \\
\text { (150mg BID) }\end{array}$ & 2 años & $1279 / 1289 / 2568$ & TVP recurrente & $\begin{array}{l}\text { Hemorragia } \\
\text { mayor }\end{array}$ \\
\hline $\begin{array}{l}\text { HOKUSAI - VTE } \\
(2013)(32)\end{array}$ & $\begin{array}{l}\text { Edoxaban } \\
\text { (60mg OD) }\end{array}$ & 2,8 años & $4118 / 4122 / 8240$ & TVP recurrente & $\begin{array}{l}\text { Hemorragia } \\
\text { mayor }\end{array}$ \\
\hline $\begin{array}{l}\text { RE-MEDY } \\
(2013)(33)\end{array}$ & $\begin{array}{l}\text { Dabigatran } \\
\text { (150mg BID) }\end{array}$ & 4 años & $1430 / 1426 / 2856$ & TVP recurrente & $\begin{array}{l}\text { Hemorraǵia } \\
\text { mayor }\end{array}$ \\
\hline
\end{tabular}

aleatorios controlados en fase III, una duración mayor de 2 años y con un número de pacientes significativo que representara una fuente confiable de evidencia científica. Los eventos primarios a analizar fueron: ictus y embolismo sistémico en FA, y Trombosis venosa recrente en el caso de TVP. Como evento a analizar en estudios de seguridad en ambas patologías se seleccionó la hemorragia mayor, calificada como la disminución del nivel de hemoglobina en $2 \mathrm{~g} / \mathrm{dL}$ en 24 horas, bajo criterios de ISTH (International Society of Thrombosis and Hemostasis) (34).

\section{Criterios para los estudios de no inferioridad}

En los ensayos de "no inferioridad", el objetivo principal es demostrar que la eficacia entre dos tratamientos es similar, uno denominado tratamiento nuevo y el otro llamado el control activo, por lo que hay una necesidad de evidencia comparativa, pero pueden aparecer sesgos de confusión por el hecho de conocer que ambos tratamientos son activos. La pregunta a hacer cuando se intenta realizar estudios de no inferioridad es: ¿El nuevo tratamiento es al menos tan eficaz como el control activo para la reducción del riesgo de eventos? De la cual pueden suceder dos respuestas: a) HO (hipótesis nula): el nuevo tratamiento es menos eficaz para la reducción de eventos (es inferior) o es más eficaz (es superior) y b) H1 (hipótesis alternativa): el nuevo tratamiento es al menos tan eficaz como el control activo para la reducción de eventos (no es inferior). Adoptar «la respuesta» $\mathrm{HO}$ como verdadera se fundamenta en una regla de decisión según la significación estadística del valor de p. Sin embargo, el valor de p que se calcula en los ensayos de no inferioridad es especial; se denomina "p de no inferioridad" diferente de la p de superioridad; la diferencia estriba en los intervalos de confianza (IC), si en el ran- 
go del IC 95\% no está incluida la unidad [1], asumimos que hay significación para superioridad (Ej.: 0,78-0,98 ó 1,08-2,23), pero si sucede lo contrario que en el rango del IC 95\% está incluida la unidad [1], se acepta que no hay significación estadística (Ej.: 0,72-1,12), y estaríamos hablando de equivalencia o no inferioridad entre dos tratamientos. Supongamos que la tasa de eventos tromboembólicos con el nuevo tratamiento es no inferior que con la warfarina (control activo), con un valor de $\mathrm{p}$ de no inferioridad $<0,001$. En este caso, adoptaremos la hipótesis alternativa H1 como cierta, porque si realmente el nuevo tratamiento fuera inferior a los antagonistas de la vitamina $\mathrm{K}$, obtener dicho resultado habría sido tan improbable como $\mathrm{p}$ $<0,001$. Los ensayos clínicos aleatorizados de no inferioridad se suelen llevar a cabo en circunstancias en que no es esperable que el tratamiento experimental sea más eficaz que el tratamiento estándar o control activo, pero el nuevo fármaco ofrece ventajas adicionales. Dichas ventajas podrían ser un mejor perfil de seguridad, menos efectos secundarios, más fácil administración, menos necesidad de controles y hasta un menor costo total $(14,35-37)$.

\section{¿Cómo se calcula el límite de no-inferioridad?}

En esta revisión sistemática se analiza la warfarina en la reducción de eventos tromboembólicos y la no inferioridad con nuevos anticoagulantes orales. Para el análisis de los estudios de no inferioridad en FA, el dintel de no inferioridad $(\Delta+)$ para la warfarina se fijó a partir de un metanálisis publicado en 1999 (38), en el que se cuantificó el efecto de la warfarina en la prevención de eventos tromboembólicos respecto al placebo o a la ausencia de tratamiento, en un riesgo relativo (RR) de 0,38 (intervalo de confianza del 95\% [IC95\%, 0,28-0,52]). Se calcula el efecto del «placebo o ausencia de tratamiento» respecto a la warfarina. En este caso, dicho efecto sería el inverso de $0,38(1 / 0,38)$, que corresponde a $\mathrm{RR}=2,63 \mathrm{y}$ el inverso del
IC95\% (IC95\%, 1,92-3,57). El margen inferior de dicho intervalo de confianza $(1,92)$, podría considerarse el dintel de no inferioridad para los nuevos anticoagulantes. Sin embargó, las aǵencias reguladoras fueron más exigentes, y eligieron un dintel de no inferioridad asumiendo que, hipotéticamente, la warfarina tuviera únicamente un 50\% del efecto que realmente tiene. Así, el dintel superior $(\Delta+)$ de no inferioridad quedaba fijado en 1,46 y el dintel inferior de los límites de no inferioridad $(\Delta-)$ quedaría en $(1-0,46=0,54)$, todo lo que rebase esos límites es demostrable para superioridad o no se demuestra no inferioridad. El límite de no inferioridad puede ser calculado de una cola, para ello el límite superior se toma del inverso del RR en un estudio previo de superioridad del control activo o bien del inverso del IC95\%, tomando en cada caso el 50\% de ambos resultados como límite superior $(\Delta+)$. Si se desea calcular los límites de no inferioridad con dos colas $(\Delta+\mathrm{y} \Delta-)$, se estaría hablando de equivalencia, el límite inferior sería $(1-\Delta+)$ que es $(1-0,46=0,54)$ $(14,35)$. Es necesario hacer la acotación que la no inferioridad se calcula en esta revisión solo en términos de efectividad, puesto que, para seguridad, la publicación seleccionada (38) para los datos primarios para calcular el límite de no inferioridad solo hace referencia al efecto de la warfarina en la prevención de ictus y no especifica muy bien los datos sobre hemorragia como evento secundario.

\section{Análisis estadístico. Descripción del metanálisis}

Se siguieron las pautas elaboradas según las recomendaciones del método PRISMA (39). Los estudios seleccionados llevaron a cabo comparaciones directas entre ACODs y warfarina sobre una base de análisis primario de eventos de efectividad y seguridad. El metanálisis se desarrolló bajo la presentación gráfica tipo diagrama de bosque o "Forest plot" con la modalidad tipo efecto aleatorio y como medida de efecto el Riesgo relativo (RR), con los componentes 
siguientes: 1. Nombre del estudio, 2. ACODs eventos/total de pacientes (\%), 3. Warfarina eventos/total de pacientes (\%), 4. Número Necesario a Tratar (NNT) para efectividad y el Número Necesario para dañar (NNH, del inglés Harm) para seguridad o efecto adverso, calculado como el inverso de la reducción o incremento absoluto del riesgo, 5 . Peso porcentual de cada estudio y 6. RR (IC95\%) de cada estudio. El análisis estadístico del metanálisis se realizó con el programa Comprehensive Meta-Analysis (Biostat, Englewood, $\mathrm{NJ}$ ). Como parámetros estadísticos se determinó el grado de heterogeneidad $\left(\mathrm{I}^{2}\right)$ en bajo $(<25 \%)$, moderado $(25 \%$ a $75 \%)$ y alto (>75\%) mediante la prueba del cálculo del chi $^{2}$ de cada metanálisis ( $Q$ de cochrane) según la fórmula de Hiǵgins (40), cálculo de $Z$ y $\mathrm{p}<0,05$ para significación estadística. Los términos significación clínica y estadística se refieren a reducción o aumento del riesgo de eventos en forma significativa o no, puede darse el caso de significación clínica con reducción o aumento de eventos sin signnificación estadística (Vizcaíno G. 2019, libro Medina Basada en la Evidencia y análisis de diseños de investigación clínica, 2 a edición, en vías de publicación).

\section{RESULTADOS}

\section{Fibrilación Auricular}

El análisis para efectividad mostró una reducción del riesǵo significativa y clínica de los ACODs sobre la warfarina, pero estuvieron dentro de los límites de no inferioridad, el resultado global muestra una reducción del riesgo de $24 \%$, con un NNT de 125 (por cada 125 pacientes tratados se previene un ACV) y un valor $\mathrm{I}^{2}$ del $0 \%$, demostrando homogeneidad con alta significación entre los estudios $(p<0,0001)$ (Fig. 1). El tiempo en el rango terapéutico (TRT) para la warfarina fue el siguiente: RE-LY: 64\%, ARISTOTLE: 66\%, ROCKET-AF: 55\% y ENGAGE-AF-TIMI 48: 68,4\%. (Fig. 1).

En el análisis de seguridad para hemorragia mayor con la administración de los ACODs versus warfarina, dos estudios (ARISTOTLE y ENGAGE-AF-TIMI 48) demostraron reducción clínica y significativa del riesǵo, mientras que los otros dos estudios (RE-LY y ROCKET-AF) también dieron resultados similares a warfarina pero no mostraron significación clínica ni estadística. Al analizar el resultado combinado demostró una reducción del riesgo de $14 \%$, pero solo se inter-

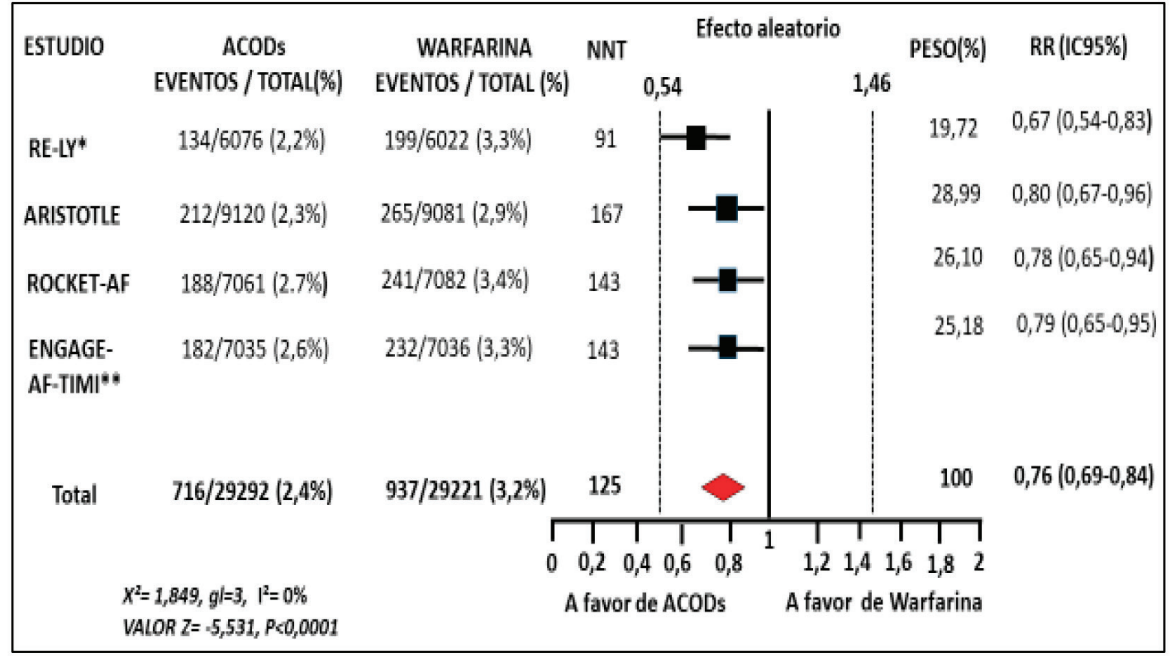

Fig. 1. Ictus y Embolismo Sistémico en Fibrilación Auricular no valvular. Estudios de no inferioridad con ACODs y warfarina como control activo.

*Dabigatran $150 \mathrm{mǵ,} \mathrm{**Edoxaban} 60 \mathrm{mǵ}$. 
pretó como reducción clínica del riesǵo, no significativa [RR (IC95\%): 0,86 (0,73-1,01), $\mathrm{p}=0,058]$. El resultado global del NNH fue de 111 (por cada 111 pacientes tratados se previene un evento hemorrágico mayor). El análisis para heterogeneidad señaló una baja heterogeneidad en el conjunto de estudios $(3,32 \%)$ (Fig. 2).

\section{Trombosis Venosa Profunda}

Como evento primario, se consideró la trombosis venosa recurrente. La figura 3 muestra el metanálisis de los seis estudios seleccionados que reportaron el efecto de los ACODs versus warfarina en TVP, la limitación encontrada al contrario de los estudios de ACODs y warfarina en FA no valvular, fue el establecer uniformemente los límites de no inferioridad, lo cual hace dificil la interpretacion de los resultados para no inferioridad cuando se estudia en conjunto el resultado del metanálisis, así vemos que el limite de no inferioridad $(\Delta+)$ fue de 1,80 para AMPLIFY; 1,5 para HOKUSAI-VTE; 2,85 para RE-MEDY y 2,0 para EINSTEIN, EINSTEIN-PE y RECOVER II.

Según el análisis realizado, todos los estudios sobre efectividad mostraron criterios de no inferioridad respecto a la warfarina, alǵunos con significación clínica pero no estadística, de reducción del riesgo (AMPLIFY, EINSTEIN y HOKUSAI-VTE), y el resto mostró incremento no significativo del riesgó (EINSTEIN-PE, RECOVER II y RE-MEDY). El resultado combinado de los estudios reveló una reducción no significativa del riesgo del 7\% [RR (IC95\%): 0,93 (0,79-1,08); $\mathrm{p}=0,372$ ] con una baja heterogeneidad $\left(\mathrm{I}^{2}=2,81 \%\right)$, el NNT fue de 500 (por cada 500 pacientes tratados se previene una recurrencia de TVP) y la distribución del peso de cada estudio según el efecto aleatorio muestra el estudio HOKUSAI-VTE con mayor peso (Fig. 3).

El análisis de seguridad (hemorragia mayor como evento a reportar), mostró superioridad en todos los estudios, de los ACODs sobre la warfarina aunque la superioridad solo se demostró significativamente en los estudios AMPLIFY y EINSTEIN-PE, pero el resultado combinado señaló que hubo una reducción relativa del riesgo de hemorragia, tanto clínica como estadística de $42 \%$ a favor de los ACODs comparados con la warfarina $[R R(I C 95 \%)=0,58(0,42-0,79), p<0,0001]$. La homogeneidad fue demostrada $\left(\mathrm{I}^{2}=0 \%\right)$ y el NNH global fue de 147 (por cada 147 pacientes tratados se previene un evento hemorrágico) (Fiǵ. 4).

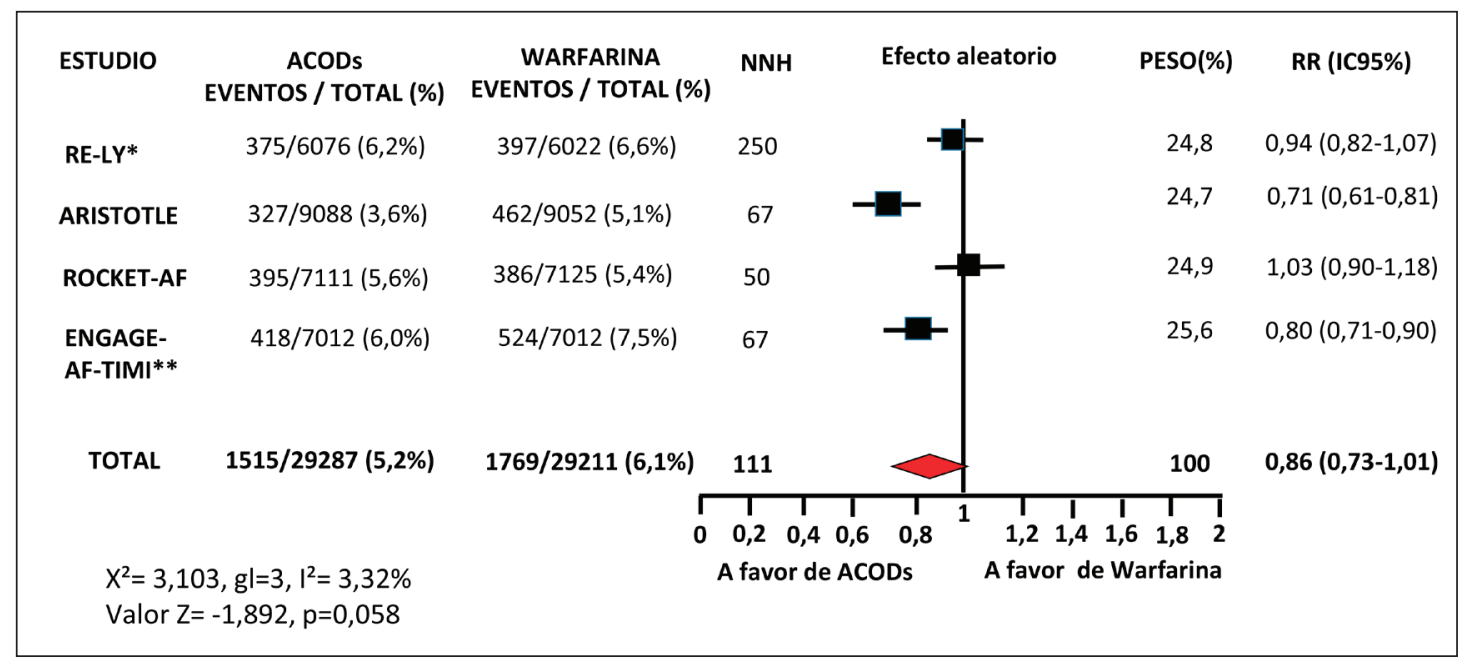

Fig. 2. Hemorragiia mayor en FA no valvular. Análisis de seguridad entre ACODs y warfarina.

*Dabigatran $150 \mathrm{mg}$, *Edoxaban 60mg.

Vol. 61(1): 73 - 90, 2020 


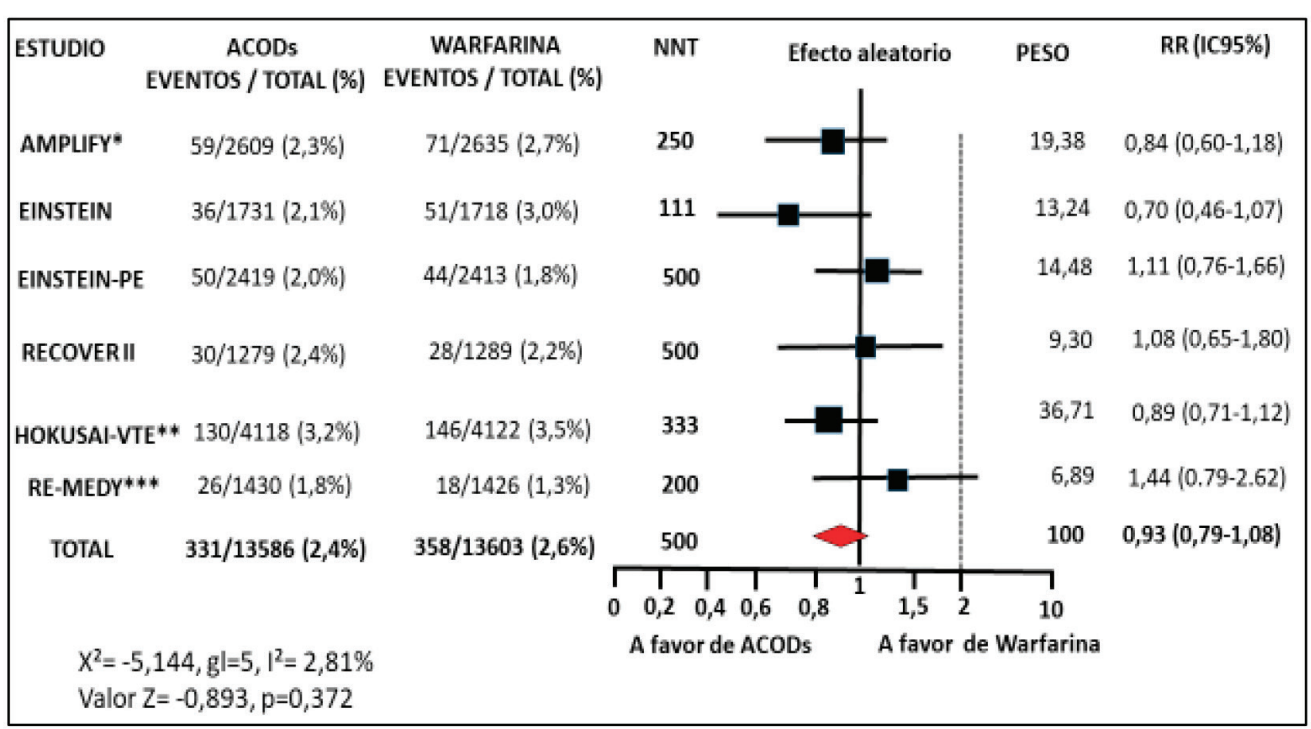

Fig. 3. Recurrencia en Tromboembolismo Venoso Profundo. Estudios de no inferioridad con ACODs y Warfarina como control activo.

"AMPLIFY el límite de no inferioridad fue 1,80; "HOKUSAI-VTE el límite de no inferioridad fue 1,5; ***RE-MEDY el límite de no inferioridad fue de 2,85.

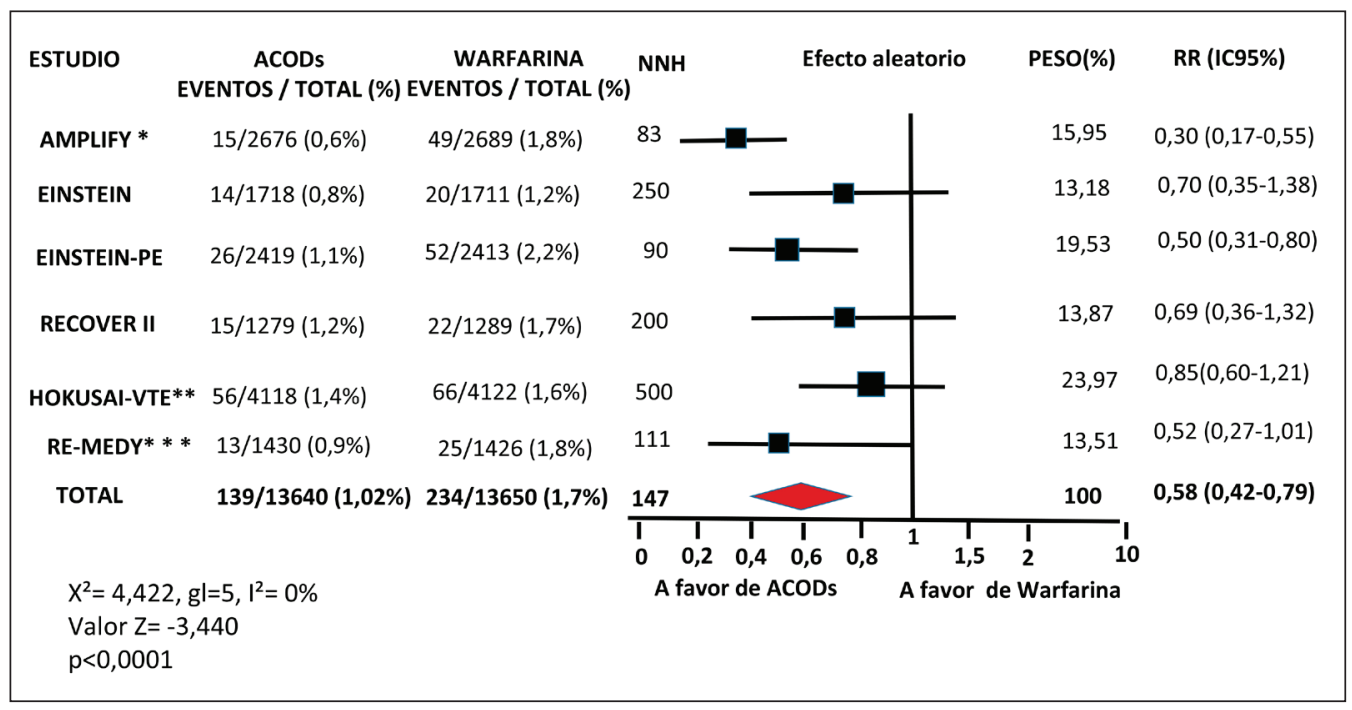

Fig. 4. Hemorragia mayor en TVP. Análisis de seguridad entre ACODs y warfarina.

"AMPLIFY el límite de no inferioridad fue 1,80; "*HOKUSAI-VTE el límite de no inferioridad fue 1,5; **:RE-MEDY el límite de no inferioridad fue de 2,85.

\section{DISGUSIÓN}

Los hallazgos en la presente revisión sistemática con estudios de metanálisis, mostraron para eficacia, que el evento primario de ictus y embolismo sistémico en FA no valvular tuvo una reducción significativa del riesgo ǵlobal de $24 \%$ con el uso de los ACODs al compararlos con la warfarina, aunque todos los estudios se reportaron como no inferioridad o equivalencia respecto a la warfarina. En el análisis de seguridad combinado no hubo diferencias significativas entre ACODs y la warfarina. Una revisión sistemá- 
tica similar (22), analizó tres estudios (RELY, ROCKET-AF y ARISTOTLE) cuyo evento primario a reportar fueron ictus isquémico y embolismo sistémico y el resultado combinado para eficacia, a diferencia de nuestros resultados, no evidenció significación $(\mathrm{RR}=$ 0,93; IC95\%=0,83-1,04), el análisis de seguridad con hemorragia mayor como evento primario tampoco reportó diferencia signnificativa con un RR de 0,86 (0,70-1,05) hallazgo similar al presente estudio. De igual manera un análisis retrospectivo sobre FA no valvular encontró que los ACODs fueron no inferiores a la warfarina en la prevención de tromboembolismo sistémico e ictus, pero causaron menos eventos hemorrágicos (41).

En relación con TVP los resultados demostraron que no hubo diferencias significativas entre los dos grupos de tratamiento, en cuanto a eficacia en la reducción de eventos de recurrencia de TVP, considerándose que todos mostraron no inferioridad de ACODs con respecto a la warfarina; pero en relación al análisis de seguridad en el resultado global combinado, se encontró una reducción signnificativa de eventos hemorrágicos a favor de los ACODs. Una revisión que combinó estudios aleatorios y observacionales en FA y TVP encontró diferencias atribuibles al diseño del estudio (mejor eficacia en los aleatorios controlados que en los observacionales), en FA el rivaroxaban tuvo una dsisminución del riesgo del 20\% en comparación con la warfarina en estudios aleatorios controlados. En cuanto a TVP recurrente los resultados fueron comparables y para seguridad los ACODs demostraron reducción de eventos hemorrágicos comparados con la warfarina entre un $32 \%$ y $69 \%$ (42).

\section{Análisis comparativo indirecto entre ACODs y warfarina}

Dado que muchas veces los resultados de los ensayos clínicos no se compaginan con la realidad, Yao y col. (43), compararon la eficacia y seguridad de cada ACODs (apixaban, rivaroxaban y dabigatran) versus warfarina, en pacientes con alto riesgo de ictus y tromboembolismo sistémico en FA no valvular $\left[\mathrm{CHA}_{2} \mathrm{DS}_{2}\right.$ VASe $\left.>2\right]$. En comparación con warfarina, el apixaban se asoció significativamente con menores riesgos para ACV y sangrado mayor $(\mathrm{p}<0,04$ y $\mathrm{p}<0,001$ respectivamente), el rivaroxaban no mostró diferencias en el riesgo de ACV y sangrado mayor y el dabigatran mostró un riesǵo similar para ACV, pero significativamente menor para sangrado mayor $(\mathrm{p}<0,01)$ (Tabla $\mathrm{V})$.

En otro estudio, Banergee y col. (44) demostraron de una forma empírica e indirectamente una eficacia similar entre ACODs y warfarina en pacientes con $\mathrm{CHADS}_{2} \geq 1 \mathrm{o}$ $\mathrm{CHA}_{2} \mathrm{DS}_{2}$-VASc $\geq 2$, con fibrilación auricular y riesgo de ictus isquémico o hemorrágico. Todos los ACODs (dabigatran, rivaroxaban y apixaban) fueron superiores a warfarina solo

TABLA V

COMPARACIÓN INDIRECTA ENTRE "GATRANES" Y "XABANES" CON WARFARINA EN LA PREVENCIÓN DE ICTUS ISQUÉMICO Y EMBOLISMO SISTÉMICO EN FIBRILACIÓN AURICULAR NO VALVULAR

\begin{tabular}{lccccc}
\hline Comparación & $\begin{array}{c}\mathrm{N}^{\circ} \text { de } \\
\text { pacientes }\end{array}$ & $\begin{array}{c}\text { Eficacia: } \\
\text { HR (IC95\%), }\end{array}$ & A favor & $\begin{array}{c}\text { Seguridad: } \\
\text { HR (IC95\%), } \mathrm{p}\end{array}$ & A favor \\
\hline Apixaban vs Warfarina & 15390 & $\begin{array}{c}0,67(0,46-0,98), \\
\mathrm{p}<0,04\end{array}$ & Apixaban & $\begin{array}{c}0,45(0,34-0,59), \\
\mathrm{p}<0,001\end{array}$ & Apixaban \\
Rivaroxaban vs warfarina & 32250 & $\begin{array}{c}0,93(0,72-1,19), \\
\mathrm{p}=0,56\end{array}$ & Ninguno & $\begin{array}{c}1,04(0,90-1,20), \\
\mathrm{p}=0,60\end{array}$ & Ninguno \\
Dabigatran vs Warfarina & 28214 & $\begin{array}{c}0,98(0,76-1,26), \\
\mathrm{p}=0,98\end{array}$ & Ninguno & $\begin{array}{c}0,79(0,67-0,94), \\
\mathrm{p}<0,01\end{array}$ & Dabigatran \\
\hline
\end{tabular}

Modificada y adaptada de Yao y col. (43).

Vol. 61(1): 73 - 90, 2020 
en el beneficio clínico neto, cuando el riesgo de hemorragia y ACV fue alto. los ACODs parecen aportar mayor beneficio, disminuyendo el riesgo de hemorragia (44). Una revision sistemática reciente (45) encontró, en un seguimiento de un año, que el apixaban (HR, 0.93; IC95\%, 0.71-1.20) y el dabigatran (HR, 0.95; IC95\%, 0.80-1.13) no mostraron diferencias en la reducción de ACV versus warfarina, mientras que el rivaroxaban si redujo significativamente el riesgo (HR, 0.83; IC95\%, 0.73-0.94). Por el contrario, en relación al sangrado mayor, el apixaban (HR, 0.66; IC95\%, 0.55-0.80) y el dabigatran (HR, 0.83 ; IC95\%, 0.70-0.97) fueron superiores a la warfarina, no así el rivaroxaban (HR, 1.02; IC95\%, 0.95-1.10); en cuanto al riesgo de hemorragia intracraneal los ACODs obtuvieron de forma significativa mayor reducción del riesgo en comparación con la warfarina (45). En pacientes asiáticos con FA, no hubo diferencia significativa entre los ACODs y la warfarina en evento primario de ictus isquémico, pero al relacionar el compuesto de ictus isquémico, hemorragia intracraneal y mortalidad por todas las causas los ACODs fueron significativamente superiores a la warfarina (46). En otro contexto el estudio REVISIT-US (47), mostró reducción de eventos de ictus isquémico y hemorrágico sobre la warfarina mientras que el apixaban fue prácticamente no inferior en efectividad y seguridad al compararse con la warfarina.

\section{Análisis comparativo entre "gatranes" y "xabanes" en Fibrilación Auricular}

Al no existir estudios comparativos ("head to head") entre los diferentes ACODs, se puede establecer una comparación indirecta entre "gatranes" y "xabanes", basada en la eficacia y seguridad demostrable en los resultados cuando se administran a pacientes con FA no valvular y prevención de ictus y embolismo sistémico. Mantha y Ansell (48) basados en los resultados de los estudios RE-LY (24), ARISTOTLE (25) y ROCKET-AF (27), señalan que hay una similitud en el efecto terapéutico entre el dabigatran (dosis de 110mǵ y 150mg), el apixaban (5mg BID) y el rivaroxaban (20mg OD) ante el riesgo de ictus y embolismo sistémico en FA no valvular a excepción de la comparación entre las dosis del dabigatran (150 $\mathrm{mg}$ ) y el rivaroxaban $(p=0,04)$ al igual que la comparación entre las dosis del dabigatran donde la dosis de $150 \mathrm{mg}$ demostró mayor efectividad que la dosis de $110 \mathrm{mg}(\mathrm{p}=0,004)$. En el caso de hemorrágia mayor, el apixaban reveló una reducción significativa de eventos sobre el dabigatran (150 mg) y el rivaroxaban. Adicionalmente, el dabigatran (110 mg) comparado con el rivaroxaban mostró significativamente menor riesgo de hemorragia (48) (Tabla VI).

En otras comparaciones indirectas entre "gatranes y xabanes" en cuanto a eficacia y seguridad para ictus en FA, solo el dabigatran $(150 \mathrm{mg})$ fue superior al rivaroxaban (20mg) (26\%), mientras que al comparar el apixaban (5mg BID) versus el dabigatran (110 mg) y el rivaroxaban no se demostraron diferencias entre ellos (49). En el mismo contexto, para la reducción de eventos hemorrágicos el apixaban resultó ser superior al dabigatran (150mg) (26\%) y el rivaroxaban (34\%). La comparación del edoxaban (60mg OD) con los otros ACODs fue la siguiente: demostró iǵual eficacia y seguridad (Hemorragia mayor) comparado con el apixaban (5mg BID); con el rivaroxaban (20mg OD) se obtuvo igual eficacia y menor riesgo de hemorragia y fue superior al dabigatran (150 mg) en eficacia y seguridad (50).

\section{Análisis comparativo entre "gatranes" y "xabanes" en Trombosis Venosa Profunda}

En la comparación indirecta sobre eficacia y seguridad entre los ACODs para TVP (Tabla VII), no se encontraron diferencias significativas entre ellos cuando se analizó la eficacia y solo el apixaban (5mg BID) demostró superioridad sobre el dabigatran $(150 \mathrm{mg}$ OD) $(\mathrm{p}<0,02)$ y el edoxaban $(60$ mg OD) $(p<0,001)$, con menor riesgo de hemorragia, en el estudio comparativo indirecto (51). 


\section{TABLA VI}

COMPARAGIÓN INDIREGTA ENTRE "GATRANES" Y "XABANES" EN LA PREVENCIÓN DE ICTUS ISQUÉMICO Y EMBOLISMO SISTÉMICO EN FIBRILACIÓN AURICULAR NO VALVULAR

\begin{tabular}{|c|c|c|c|c|}
\hline Comparación & $\begin{array}{l}\text { Eficacia: Ictus, } \\
\text { OR (IC95\%), p }\end{array}$ & A Favor & $\begin{array}{l}\text { Seguridad: } \\
\text { Hemorraǵia } \\
\text { OR (IC95\%) p }\end{array}$ & A Favor \\
\hline $\begin{array}{l}\text { Apixaban ( } 5 \mathrm{mg} \text { BID) } \\
\text { vs Dabigatran (150mg OD) }\end{array}$ & $\begin{array}{l}1,22(0,91-1,62) \\
p=0,18\end{array}$ & Ninǵuno & $\begin{array}{l}0,74(0,61-0,91) \\
\mathrm{p}<0,004\end{array}$ & Apixaban \\
\hline $\begin{array}{l}\text { Apixaban ( } 5 \mathrm{mg} \text { BID) } \\
\text { vs Dabigatran (110mg OD) }\end{array}$ & $\begin{array}{l}0,88(0,67-1,15) \\
\mathrm{p}=0,34\end{array}$ & Ninguno & $\begin{array}{l}0,87(0,70-1,06) \\
\mathrm{p}=0,17\end{array}$ & Ninǵuno \\
\hline $\begin{array}{l}\text { Apixaban (5mg BID) } \\
\text { vs Rivaroxaban ( } 20 \mathrm{mg} \text { OD) }\end{array}$ & $\begin{array}{l}0,90(0,71-1,16) \\
\mathrm{p}=0,43\end{array}$ & Ninǵuno & $\begin{array}{l}0,68(0,55-0,83) \\
\mathrm{p}<0,001\end{array}$ & Apixaban \\
\hline $\begin{array}{l}\text { Rivaroxaban (20mg OD) } \\
\text { vs Dabigatran (150mg OD) }\end{array}$ & $\begin{array}{l}1,35(1,02-1,78) \\
\mathrm{p}<0,04\end{array}$ & Dabigatran & $\begin{array}{l}1,10(0,90-1,34) \\
p=0,36\end{array}$ & Ninǵuno \\
\hline $\begin{array}{l}\text { Rivaroxaban (20mg OD) } \\
\text { vs Dabigatran (110mg OD) }\end{array}$ & $\begin{array}{l}0,97(0,74-1,26) \\
p=0,81\end{array}$ & Ninǵuno & $\begin{array}{l}1,28(1,04-1,57) \\
\mathrm{p}<0,02\end{array}$ & Dabigatran \\
\hline \multicolumn{5}{|c|}{$\begin{array}{l}\text { Modificada y adaptada de: Mantha S, Ansell J. (47). Datos tomados de la combinación de los estudios RE-LY (24) } \\
\text { ARISTOTLE (25) y ROCKET-AF (26). }\end{array}$} \\
\hline \multicolumn{5}{|c|}{$\begin{array}{c}\text { TABLA VII } \\
\text { COMPARACIÓN INDIRECTA ENTRE "GATRANES" Y "XABANES" EN LA PREVENCIÓN } \\
\text { DE TROMBOSIS VENOSA PROFUNDA RECURRENTE }\end{array}$} \\
\hline Comparación & $\begin{array}{l}\text { Eficacia: } \\
\text { RR (IC95\%), p }\end{array}$ & A Favor & $\begin{array}{l}\text { Seguridad } \\
\text { RR (IC95\%), p }\end{array}$ & A Favor \\
\hline Apixaban vs Dabigatran & $\begin{array}{l}0,77(0,47-1,27) \\
\mathrm{p}=0,30)\end{array}$ & Ningúno & $\begin{array}{l}0,42(0,21-0,87) \\
\mathrm{p}<0,02\end{array}$ & Apixaban \\
\hline Apixaban vs Rivaroxaban & $\begin{array}{l}0,94(0,60-1,48) \\
\mathrm{p}=0,80\end{array}$ & Ninguno & $\begin{array}{l}0,57(0,29-1,15) \\
\mathrm{p}=0,12\end{array}$ & Ninguno \\
\hline Apixaban vs Edoxaban & $\begin{array}{l}0,94(0,62-1,43) \\
\mathrm{p}=0,78\end{array}$ & Ninǵuno & $\begin{array}{l}0,37(0,19-0,73) \\
\mathrm{p}<0,001\end{array}$ & Apixaban \\
\hline Rivaroxaban vs Dabigatran & $\begin{array}{l}0,82(0,51-1,30) \\
\mathrm{p}=0,40\end{array}$ & Ninǵuno & $\begin{array}{l}0,74(0,42-1,30) \\
\mathrm{p}=0,30\end{array}$ & Ninguno \\
\hline Rivaroxaban vs Edoxaban & $\begin{array}{l}1,00(0,68-1,46) \\
\mathrm{p}=0,99\end{array}$ & Ninǵuno & $\begin{array}{l}0,64(0,38-1,08) \\
\mathrm{p}=0,10\end{array}$ & Ninguno \\
\hline Edoxaban vs Dabigatran & $\begin{array}{l}0,82(0,53-1,26) \\
\mathrm{p}=0,36\end{array}$ & Ninǵuno & $\begin{array}{l}1,15(0,66-2,00) \\
\mathrm{p}=0,62\end{array}$ & Ninguno \\
\hline
\end{tabular}

Modificada y adaptada de Mantha S, Ansell J (51). Datos tomados de la combinación de los estudios AMPLIFY (28), EINSTEIN (29), EINSTEIN-PE (30), RECOVER II (31) y HOKUSAI-VTE (32).

La controversia entre los ACODs y la warfarina como tratamiento preferencial en FA persiste, a pesar de una demostrable eficacia y seguridad de los ACODs sobre la warfarina, y hay ciertos aspectos que no han sido suficientemente aclarados o los estudios no son concluyentes. Ansell (52) mencionó ciertas desventajas del uso de ACODs ante la warfarina como tratamiento inicial, sobre warfarina entre las que destacan su media vida más corta, la falta de control de la coagulación y precisión del efecto anticoagulan- 
te en el laboratorio, y su alto costo; todo ello podría conducir a falta de adherencia del paciente al tratamiento anticoagulante. Por el contrario, Granger y Armaganijan (53) señalaron algunas ventajas con el uso de los ACODs en lugar de warfarina en FA, demostrables por la baja frecuencia de ictus, menor tasa de hemorragia y menor tasa de mortalidad que se evidenciaron en grandes ensayos aleatorios controlados y con alto poder estadístico. Todo ello, pros y contras sobre el uso de anticoagulación en FA determinan que el médico generalmente conservador, piense seriamente a la hora de prescribir estos medicamentos, en especial si es un paciente de edad, con compromiso renal o con antecedentes de episodios hemorrágicos (54).

Un estudio sobre efectividad y seguridad entre ACODs y warfarina en FA y TVP concluyó que los ACODs son una opción viable en pacientes con anticoagulación a largoo plazo, porque poseen una anticoagulación más predecible, menor interacción con otras drogas y similar o menor índice de mortalidad y eventos adversos (55). Cuando no es posible seguir un control estricto de los pacientes que reciben warfarina, con pobre control del INR, los ACODs (dabigatran) demostraron ser superiores en la reducción de eventos sobre eficacia y seguridad (56). En relación a los análisis económicos, es probable que el apixaban sea particularmente rentable en pacientes con mayor riesgo de ACV o en aquellos pacientes donde el INR es menos controlado o es difícil de estabilizar, sin embargo, un nuevo agente puede no ser necesario para los pacientes en los que el INR ha sido bien controlado con warfarina (57). En cuanto a los ACODs, quedan importantes preguntas con respecto a sus respectivos perfiles de eficacia y seguridad, especialmente en la comparación entre ellos (57).

Esta revisión sistemática presenta varias limitaciones. El análisis de la efectividad en los diferentes estudios fue preespecificado solo para eventos primarios como ictus isquémico y embolismo sistémico en FA por su mayor frecuencia como evento a reportar y para TVP se analizó como evento primario la recurrencia de trombosis venosa, no hubo análisis de eventos primarios compuestos o eventos secundarios en ambas patologías. Para unificar la medida de efecto en el metanálisis se escogió el riesgo relativo (RR) a pesar que algunos estudios han reportado como medida de efecto hazard ratio o diferencia porcentual. Por otra parte, en el análisis de seguridad solo se estudiaron eventos de hemorragia mayor, los eventos reportados como hemorragia no mayor clínicamente relevante, no fueron analizados. Otro aspecto a diferenciar es el diseño del ensayo clínico, por ejemplo: El rivaroxaban fue superior a la warfarina en el diseño por protocolo ("as treated"), y fue no inferior en el diseño de intención de tratar (Intention to treat) (25). En relación a los diferentes estudios, se escogió una sola dosis para cada ensayo clínico, es así que para el dabigatran se escogió la dosis de $150 \mathrm{mg}$ que es la de mayor efectividad, y tiene una tasa de eventos hemorráǵicos similar a la dosis de $110 \mathrm{mg}$, además de que se reporta controversia en el uso de esta última dosis y no fue aprobada en los Estados Unidos $(42,43)$. Los estudios con edoxaban se analizaron solo con la dosis administrada de $60 \mathrm{mg}$ y no se estudiaron pacientes que recibieron $30 \mathrm{mg}$, ambos regímenes de una vez al día de este medicamento, fueron no inferiores a la warfarina para la prevención de ACV o embolia sistémica y se asociaron con tasas significativamente más bajas de sangrado y muerte por causas cardiovasculares $(27,58)$. Algunos aspectos dentro del tratamiento anticoagulante en TVP, no fueron considerados en esta revisión sistemática, como el manejo de la hemorragia en el paciente anticoagulado con ACODs o warfarina, para lo cual hacemos referencia a las guías de la Asociación Americana de Hematología (ASH) (59). Otro aspecto limitante que tampoco fue considerado fue el uso de anticoagulantes y warfarina en relación con el peso corporal, respecto a ello, en un estudio de pos hoc del ARISTOTLE (60), el apixaban resultó con mayor efectividad (HR 
0,71, IC 95\% 0,61-0,83) y seguridad (HR 0,71 , IC 95\% 0,61-0,83) que la warfarina en pacientes con peso normal y en obesos.

La decisión de utilizar el tratamiento anticoagulante, bien sea ACODs o warfarina, depende del juicio crítico del médico y del balance beneficio/riesgo para el paciente. Un estudio de cohorte poblacional de atención de rutina indicó una efectividad y seguridad similares o mejores con los ACODs, en comparación con el tratamiento con la warfarina y los primeros se asociaron con menos hemorragias intracraneales, pero más hemorragias gastrointestinales (61).

En conclusión, estos resultados señalan que los ACODs son una buena alternativa para el uso terapéutico en FA y TVP, con una eficacia similar a la warfarina pero una ligera ventaja en la reducción del riesgo hemorrágico.

\section{REFERENCIAS}

1. Pirmohamed M. Warfarin: almost 60 years old and still causing problems. Br J Phamacol 2006;62:509-511.

2. Quintero González JA. Cincuenta años de uso clínico de la warfarina. Invest Clin 2010;51:269-287.

3. Pérez Requejo JL, Martínez Gutiérrez E, Castillo González E, Ettedgui de Pérez G. Nuevos paradiǵmas en anticoagulación oral. http://sveardiologia.org/es/index. php/publicaciones/e-books/463, 2016.

4. Quintero J, Torres E, Diez-Ewald M, Arteaga-Vizeaíno M, Vizeaíno G, León M, Rodríguez Z, Fernández N. Uso de warfarina y bajo rango de INR en la prevención de trombosis venosa recurrente. Invest Clin 2011;52:230-238.

5. Ridker PM, Goldhaber SZ, Danielson E, Rosenberg Y, Eby CS, Deitcher SR, Cushman M, Moll S, Kessler CM, Elliott CG, Paulson R, Wong T, Bauer KA, Schwartz BA, Miletich JP, Bounameaux H, Glynn RJ; PREVENT Investigators. Long-term, low-intensity warfarin therapy for the prevention of recurrent venous thromboembolism. N Enǵl J Med 2003;348:1425-1434.
6. Kearon C, Ginsberg JS, Kovacs MJ, Anderson DR, Wells P, Julian JA, MacKinnon B, Weitz JI, Crowther MA, Dolan S, Turpie AG, Geerts W, Solymoss S, van Nguyen P, Demers C, Kahn SR, Kassis J, Rodger M, Hambleton J, Gent M; Extended Low-Intensity Anticoagulation for Thrombo-Embolism Investigators. Comparison of low-intensity warfarin therapy with conventional-intensity warfarin therapy for long-term prevention of recurrent venous thromboembolism. N Enǵl J Med 2003;349:631-639.

7. Pengo V. Management of oral anticoagulant treatment in patients with venous thromboembolism. Semin Thromb Hemost 2006;32:781-786.

8. Van Spall HGC, Salim Yusuf LW, Eikelboom JW, Nieuwlaat $R$, Yang $S$, Kabali $C$, Reilly PA, Ezekowitz MD, Connolly SJ. Variation in warfarin dose adjustment practice is responsible for differences in the quality of anticoagulation control between centers and countries. An analysis of patients receiving warfarin in the Randomized Evaluation of Long-Term Anticoagulation Therapy (RELY) Trial. Circulation 2012;126:2309-2316.

9. Vestergaard AS, Skjøth F, Larsen TB, Ehlers LH. The importance of mean time in therapeutic range for complication rates in warfarin therapy of patients with atrial fibrillation: A systematic review and meta-regression analysis. PLoS One 2017;12(11):e0188482. doi: 10.1371/journal.pone.0188482.

10. Xiong Q, Lau YC, Lip GY. Pharmacodynamic profile and drug interactions with nonvitamin $\mathrm{K}$ antagonist oral anticoagulants: implications for patients with atrial fibrillation. Expert Opin Drug Metab Toxicol 2015;11:937-948.

11. Hellwig T, Gulseth M. Pharmacokinetic and pharmacodynamic drug interactions with new oral anticoagulants: what do they mean for patients with atrial fibrillation? Ann Pharmacother 2013;47:1478-1487.

12. Hylek EM. Anticoagulation therapy for atrial fibrillation. Semin Thromb Hemost 2013;39:147-152.

13. Ahrens I, Lip GY, Peter K. New oral anticoagulant drugs in cardiovascular disease. Thromb Haemost 2010;104:49-60.

Vol. 61(1): 73 - 90, 2020 
14. Ferreira-González I. Bases para la interpretación de los estudios de no inferioridad: a propósito de los estudios ROCKETAF, RE-LY y ARISTOTLE. Rev Esp Cardiol 2014;67(6):432-435.

15. ICH Expert Working Group (1998) Statistical principles for clinical trials E9.Available from www.ich.org.

16. Committee for Medicinal Products for $\mathrm{Hu}$ man Use (CHMP). Guideline on the choice of the non-inferiority margin. Stat Med 2006;25(10):1628-1638.

17. Committee for Proprietary Medicinal Products (CPMP). Points to consider on switching between superiority and non-inferiority. (2000). Available from https://www. ema.europa.eu. CPMP/EWP/482/99.

18. Hylek EM, Go AS, Chang Y, Jensvold NG, Henault LE, Selby JV, Singer DE. Effect of intensity of oral anticoaǵulation on stroke severity and mortality in atrial fibrillation. N Engl J Med 2003;349:1019-1026.

19. Kearon C, Akl EA, Comerota AJ, Prandoni P, Bounameaux H, Goldhaber SZ, Nelson ME, Wells PS, Gould MK, Dentali F, Crowther M, Kahn SR. Antithrombotic therapy for VTE disease: antithrombotic therapy and prevention of thrombosis, 9th ed: American College of Chest Physicians, Evidence-Based Clinical Practice Guidelines. Chest 2012;141(2 Suppl):e419Se496S. doi: 10.1378/chest.11-2301.

20. Ageno W, Gallus AS, Wittkowsky A, Crowther M, Hylek EM, Palareti G. Oral anticoagulant therapy: antithrombotic therapy and prevention of thrombosis, 9th ed: American College of Chest Physicians, Evidence-Based Clinical Practice Guidelines. Chest. 2012;141(2 Suppl):e44S-e88S. doi: 10.1378/chest.11-2292.

21. Dentali F, Riva N, Crowther M, Turpie AGG, Lip GYH, Ageno W. Efficacy and safety of the novel oral anticoagulants in atrial fibrillation. A systematic review and meta-analysis of the literature. Circulation 2012;126:2381-2391.

22. Gómez-Outes A, Terleira-Fernández AI, Calvo-Rojas G, Suárez-Gea MA, VarǵasCastrillón E. Dabigatran, rivaroxaban, or apixaban versus warfarin in patients with nonvalvular atrial fibrillation: a systematic review and meta-analysis of subgroups.
Thrombosis 2013;2013:640723. doi: 10.1 $155 / 2013 / 640723$.

23. Fox BD, Kahn SR, Langleben D, Eisenberg MJ, Shimony A. Efficacy and safety of novel oral anticoagulants for treatment of acute venous thromboembolism: direct and adjusted indirect meta-analysis of randomised controlled trials. BMJ 2012;345:e7498, doi:10.1136/bmj.e7498.

24. Connolly, SJ, Ezekowitz MD, Yusuf S, Reilly PA, Wallentin L. Newly identified events in the RE-LY Trial. N Engl J Med 2010;363(19):1875-1876.

25. Granger CB, Alexander JH, McMurray JJ, Lopes RD, Hylek EM, Hanna M, AlKhalidi HR, Ansell J, Atar D, Avezum A, Bahit MC, Diaz R, Easton JD, Ezekowitz JA, Flaker G, Garcia D, Geraldes M, Gersh BJ, Golitsyn S, Goto S, Hermosillo AG, Hohnloser SH, Horowitz J, Mohan P, Jansky P, Lewis BS, Lopez-Sendon JL, Pais P, Parkhomenko A, Verheugt FW, Zhu J, Wallentin L; ARISTOTLE Committees and Investigators. Apixaban versus warfarin in patients with atrial fibrillation. N Engl J Med 2011;365:981-992.

26. Patel MR, Mahaffey KW, Garg J, Pan G, Singer DE, Hacke W, Breithardt G, Halperin JL, Hankey GJ, Piccini JP, Becker RC, Nessel CC, Paolini JF, Berkowitz SD, Fox KA, Califf RM; ROCKET AF Investigators. Rivaroxaban versus warfarin in nonvalvular atrial fibrillation. $\mathrm{N}$ Enǵl $\mathrm{J}$ Med 2011;365:883-891.

27. Giugliano RP, Ruff CT, Braunwald E, Murphy SA, Wiviott SD, Halperin JL, Waldo AL, Ezekowitz MD, Weitz JI, Špinar J, Ruzyllo W, Ruda M, Koretsune Y, Betcher J, Shi M, Grip LT, Patel SP, Patel I, Hanyok JJ, Mercuri M, Antman EM; ENGAGE AF-TIMI 48 Investigators. Edoxaban versus warfarin in patients with atrial fibrillation. N Engl J Med 2013;369:2093-2104.

28. Agnelli G, Buller HR, Cohen A, Curto M, Gallus AS, Johnson M, Masiukiewicz U, Pak R, Thompson J, Raskob GE, Weitz JI; AMPLIFY Investigators. Oral apixaban for the treatment of acute venous thromboembolism. N Engl J Med 2013;369:799-808

29. The EINSTEIN Investigators. Oral rivaroxaban for symptomatic venous thromboembolism. N Enǵl J Med 2010;363:2499-2510. 
30. The EINSTEIN-PE Investigators. Oral rivaroxaban for the treatment of symptomatic pulmonary embolism. N Enǵl J Med 2012;366:1287-1297.

31. Schulman S, Kakkar AK, Goldhaber SZ, Schellong S, Eriksson H, Mismetti P, Christiansen AV, Friedman J, Le Maulf F, Peter N, Kearon C; RE-COVER II Trial Investigators. Treatment of acute venous thromboembolism with dabigatran or warfarin and pooled analysis. Circulation 2013;129:764-772.

32. The Hokusai-VTE Investigators. Edoxaban versus warfarin for the treatment of symptomatic venous thromboembolism. N Engl J Med 2013;369:1406-1415.

33. Schulman S, Kearon C, Kakkar AK, Schellong S, Eriksson H, Baanstra D, Kvamme AM, Friedman J, Mismetti P, Goldhaber SZ; RE-MEDY Trial Investigators; RESONATE Trial Investigators. Extended use of dabigatran, warfarin, or placebo in venous thromboembolism. N Enǵl J Med 2013;368:709-718.

34. Schulman S, Kearon C. Subcommittee on Control of Anticoagulation of the Scientific and Standardization Committee of the International Society on Thrombosis and Haemostasis. Definition of major bleeding in clinical investigations of antihemostatic medicinal products in non-surgical patients. J Thromb Haemost 2005;3:692-694.

35. Vizcaíno G. La Estadística al alcance del Médico. Análisis crítico de ensayos clínicos. Capítulo III. Los estudios de no inferioridad en clínica médica. Ediciones Astro Data, ISBN 978-980-402-254-5, Maracaibo, pp 153.

36. Hung HM, Wang SJ, Tsong Y, Lawrence J, O'neil RT. Some fundamental issues with non-inferiority testing in active controlled trials. Stat Med 2003;22:213-225.

37. Pluchino S. Estudios clínicos de no inferioridad y de equivalencia: metodología, análisis e interpretación de los resultados. Avances Cardiol 2009; 29:76-81.

38. Hart RG, Benavente O, McBride R, Pearce LA. Antithrombotic therapy to prevent stroke in patients with atrial fibrillation: a meta-analysis. Ann Intern Med 1999;131:492-501.
39. Moher D, Liberati A, Tetzlaff J, Altman DG; PRISMA Group. Preferred reporting items for systematic reviews and metaanalyses: the PRISMA statement. PLoS Med 2009;6(7):e1000097. doi: 10.1371/journal.pmed.1000097.

40. Hig̉gins JPT, Thompson SG, Deeks JJ, Altman DG. Measuring inconsistency in metaanalyses. BMJ 2003;327:557-560.

41. Sjögren V, Byström B, Renlund H, Svensson PJ, Oldgren J, Norrving B, Själander A. Non-vitamin $\mathrm{K}$ oral anticoaǵulants are non-inferior for stroke prevention but cause fewer major bleedings than well-managed warfarin: A retrospective register study. PLoS One 2017;12(7): e0181000. Published online $2017 \mathrm{Jul} 10$. doi: 10.1371/journal.pone.018100.

42. Almutairi AR, Zhou L, Gellad WF, Lee JK, Slack MK, Martin JR, Lo-Ciganic WH. Effectiveness and safety of non-vitamin $\mathrm{K}$ antaǵonist oral anticoagulants for atrial fibrillation and venous thromboembolism: a systematic review and meta-analyses. Clin Ther 2017;39(7):1456-1478.

43. Yao X, Abraham NS, Sangaralingham LR, Bellolio F, McBane RD, Shah ND, Noseworthy PA. Effectiveness and safety of dabigatran, rivaroxaban, and apixaban versus warfarin in non-valvular atrial fibrillation. J Am Heart Assoc 2016;5:e003725 doi: 10.1161/ JАHA.116.003725.

44. Banerjee A, Lane DA, Torp-Pedersen C, Lip GY. Net clinical benefit of new oral anticoaǵulants (dabiǵatran, rivaroxaban, apixaban) versus no treatment in a 'real world' atrial fibrillation population: A modelling analysis based on a nationwide cohort study. Thromb Haemost 2012;107:584-589.

45. Escobar C, Martí-Almor J, Pérez Cabeza A, Martínez-Zapata MJ. Direct oral anticoaǵulants versus vitamin $\mathrm{K}$ antagonists in real-life patients with atrial fibrillation. A systematic review and meta-analysis. Rev Esp Cardiol (Engl Ed). 2019;72(4):305-316.

46. Cha MJ, Choi EK, Han KD, Lee SR,Lim WH, Oh S, Lip GY. Effectiveness and safety of non-vitamin $\mathrm{K}$ antagonist oral anticoaǵulants in Asian patients with atrial fibrillation. Stroke 2017;48:00-00. Doi: 10.1161/ STROKEAHA.117.018773. 
47. Coleman CI, Antz M, Bowrin K, Evers T, Simard EP, Bonnemeier H, Cappato R. Real-world evidence of stroke prevention in patients with non valvular atrial fibrillation in the United States: the REVISIT-US Study. Curr Med Res Opin 2016;32:2047-2053.

48. Mantha S, Ansell J. An indirect comparison of dabigatran, rivaroxaban and apixaban for atrial fibrillation. Thromb Haemost 2012;108:476-484.

49. Lip GY, Larsen TB, Skjøth F, Rasmussen LH. Indirect comparisons of new oral anticoaǵulant drugs for efficacy and safety when used for stroke Prevention in Atrial Fibrillation. J Am Coll Cardiol 2012;60:738-746.

50. Skjøth F, Larsen TB, Rasmussen LH, Lip GY. Efficacy and safety of edoxaban in comparison with dabigatran, rivaroxaban and apixaban for stroke prevention in atrial fibrillation. Thromb Haemost 2014;111: 981-998.

51. Mantha S, Ansell J. Indirect comparison of dabigatran, rivaroxaban, apixaban and edoxaban for the treatment of acute venous thromboembolism. J Thromb Thrombolysis 2015;39:155-165.

52. Ansell J. New oral anticoagulants should not be used as first-line agents to prevent thromboembolism in patients with atrial fibrillation. Circulation 2012;125:165-170.

53. Granger CB, Armaganijan LV. Newer oral anticoagulants should be used as first-line agents to prevent thromboembolism in patients with atrial fibrillation and risk factors for stroke or thromboembolism. Circulation 2012;125:159-164.

54. Goldhaber SZ. What's the "go to" anticoaǵulant for stroke prevention in atrial fibrillation? Thromb Haemost 2012;107(03):397-398.

55. Adam SS, MeDuffie JR, Ortel TL, Williams Jr JW. Comparative effectiveness of warfarin and new oral anticoagulants for the management of atrial fibrillation and venous thromboembolism. A systematic review. Ann Intern Med 2012;157:796-807.

56. Wallentin L, Yusuf S, Ezekowitz MD, Alings M, Flather M, Franzosi MG, Pais P, Dans A, Eikelboom J, Oldgren J, Pogue
J, Reilly PA, Yang S, Connolly SJ; RE-LY investigators. Efficacy and safety of dabigatran compared with warfarin at different levels of international normalised ratio control for stroke prevention in atrial fibrillation: an analysis of the RE-LY trial. Lancet 2010;376: 975-983.

57. Nedeltche K. Critique of apixaban versus warfarin in patients with atrial fibrillation. Stroke 2012;43:922-923, doi: 10.1161/ STROKEAHA.111.640961.

58. Goette A, Merino JL, Ezekowitz MD, Zamoryakhin D, Melino M, Jin J, Mercuri MF, Grosso MA, Fernandez V, Al-Saady N, Pelekh N, Merkely B, Zenin S, Kushnir M, Spinar J, Batushkin V, de Groot JR, Lip GY; ENSURE-AF investigators. Edoxaban versus enoxaparin-warfarin in patients undergoing cardioversion of atrial fibrillation (ENSURE-AF): A randomised, open-label, phase $3 \mathrm{~b}$ trial. Lancet 2016;388(10055):1995-2003.

59. Witt DM, Nieuwlaat R, Clark NP, Ansell J, Holbrook A, Skov J, Shehab N, Mock J, Myers T, Dentali F, Crowther MA, Agarwal A, Bhatt M, Khatib R, Riva JJ, Zhang Y, Guyatt G. American Society of Hematology 2018 guidelines for management of venous thromboembolism: optimal management of anticoagulation therapy. Blood Adv 2018;2(22):3257-3291.

60. Hohnloser SH, Fudim M, Alexander JH, Wojdyla DM, Ezekowitz JA, Hanna M, Atar D, Hijazi Z, Bahit MC, Al-Khatib SM, Lopez-Sendon J, Wallentin L, Granger CB, Lopes RD. Efficacy and safety of apixaban versus warfarin in patients with atrial fibrillation and extremes in body weight: insights from the ARISTOTLE Trial. Circulation. 2019;139:2292-2300.

61. Forslund T, Wettermark B, Andersen M, Hjemdahl P. Stroke and bleeding with non-vitamin $\mathrm{K}$ antagonist oral anticoagulant or warfarin treatment in patients with non-valvular atrial fibrillation: a population-based cohort study. Europace 2018;20(3):420-428. 